Ann. Zootech., I980, 29 (4), 377-385.

\title{
Évaluation chez le poney de la digestibilité des pellicules de soja, du marc de pommes, des caroubes et du tourteau de pépins de raisins
}

\author{
R. WOLTER $\left({ }^{*}\right)$, Andrée DURIX, J. C. LETOURNEAU et Monique CARCELEN
}

avec la collaboration technique de A. BRUNy et de A. VILLARD

Laboratoive de Nutrition et d'Alimentation

I.N.R.A., École Nationale Vétérinaive de Lyon,

Marcy l'Étoile, 69z6o Charbonnières (France)

\section{Résumé}

Quatre aliments : pellicules de soja, marc de pommes, caroubes et tourteau de pépins de raisins, ont été distribués sans conditionnement, à raison de $2 \mathrm{~kg} /$ animal/jour, à 6 ou 4 poneys placés en cages à métabolisme, afin d'en déterminer la digestibilité totale.

Les taux de glucides membranaires évalués par les méthodes de Weenđe, Scharrer ou Van Soest sont similaires, excepté pour le tourteau de pépins de raisins qui est particulièrement riche en lignine.

Ces essais font ressortir la meilleure valeur alimentaire des pellicules de soja, comparables aux pulpes đe betteraves, alors que caroube et marc de pommes se rapprochent du son de blé et de la farine de luzerne. Ils mettent en évidence la nette infériorité du tourteau de pépins de raisins dont le taux de lignine déprime très nettement la digestibilité.

\section{Introduction}

En vue d'apprécier la valeur alimentaire des différentes matières premières utilisées, ou susceptibles de l'être, dans l'alimentation des chevaux, nous avons d'abord déterminé la digestibilité d'un foin de prairie naturelle, présenté sous forme longue, broyée ou agglomérée (WOLTER, DURIX et LETOURNEAU, 1974 et I975), puis d'aliments complets, en farine, en granulés ou expansés (WOL,TER et al., I976), parallèlement à des études de la digestion de ces mêmes aliments chez des poneys fistulés (WOLTER et al., r978) ou chez des équidés abattus (Wol,TER \& GouY,

$\left(^{*}\right)$ Adresse actuelle : Chaire de Nutrition et d'Alimentation, Licole nationale vétérinaire d'Alfort. 7, avenue du Général-de-Gaulle, 94704 Maisons-Alfort Cedex. 
I976; Wolter \& ChaAbouni, 1979). Nous avons également comparé la digestibilité du maïs-fourrage déshydraté, des pulpes sèches de betteraves, de la luzerne déshydratée, du son de blé, de la paille de blé et des pulpes de raisins (Wor,TER et al., I 979). Nous rapportons à présent la poursuite de ces essais concernant les pellicules de graines de soja, les gousses dénoyautées de caroubes, le marc de pomımes, et le tourteau de pépins de raisins.

\section{I. - Conditions expérimentales}

\section{A) Animaux et aliments}

Selon un protocole déjà décrit (WoLTER et al., I979) six poneys, âgés de 7 ans et pesant $190 \mathrm{~kg}$ en moyenne, sont placés en cages à digestibilité, et reçoivent de façon exclusive chacun des aliments à raison de $2 \mathrm{~kg} / \mathrm{j}$, en 2 repas égaux distribués à 8 heures et à $\mathrm{I} 6$ heures, pendant des périodes successives comportant 3 semaines d'adaptation et 6 jours de récolte des matières fécales.

Ces aliments, dont la composition chimique est rapportée au tableau I, sont présentés sans conditionnement, c'est-à-dire en farine plus ou moins grossière. I'appétence apparaît excellente pour les pellicules de soja, satisfaisante pour les caroubes, mais insuffisante pour le marc de pommes et le tourteau de pépins de raisins qui ont été refusés respectivement par 2 animaux ou par l'un d'entre eux.

\section{B) Méthodes d'analyses}

Les prélèvements aliquotes des récoltes quotidiennes totales de matières fécales sont vaporisés avec une solution diluée d'acide sulfurique à 3 p. Ioo afin de limiter les pertes d'azote volatil; ils sont ensuite séchés à $80^{\circ} \mathrm{C}$ pendant 48 heures, puis homogénéisés, avant d'être soumis aux analyses suivantes :

- protéines totales $(\mathrm{N} \times 6,25)$, par la méthode de Kjeldah1;

- cellulose brute, par les techniques de Weende et de Scharrer;

- constituants membranaires, dosés selon Van Soest: fibres totales (N.D.F.), lignocellulose (A.D.F.) et lignine, ou déduits par différence: bémicelluloses (N.D.F. - A.D.F. ) et cellulose " vraie " (lignocellulose — lignine);

- matières minérales totales, par incinération à $55^{\circ}{ }^{\circ} \mathrm{C}$ pendant 5 heures;

- calcium, par photométrie de flamme;

- phosphore, par colorimétrie.

Parallèlement aux mesures de digestibilité, nous avons suivi l'évolution postprandiale de la glycémie, capable de traduire quelque peu l'intensité de la résorption intestinale de glucose à partir de l'extractif non azoté facilement digestible. Dans ce but, du sang jugulaire est prélevé dans des tubes héparinés juste avant le repas du matin, puis $2-4$ et 8 heures après celui-ci; le plasma est immédiatement séparé par centrifugation et rapidement congelé. Le taux de glucose est ensuite déterminé au moyen de la glucose-oxydase-peroxydase sur appareil automatique Technicon (MICHEL, I97I). 
“ DIGESTIBILITÉ CHEZ LE PONEY "

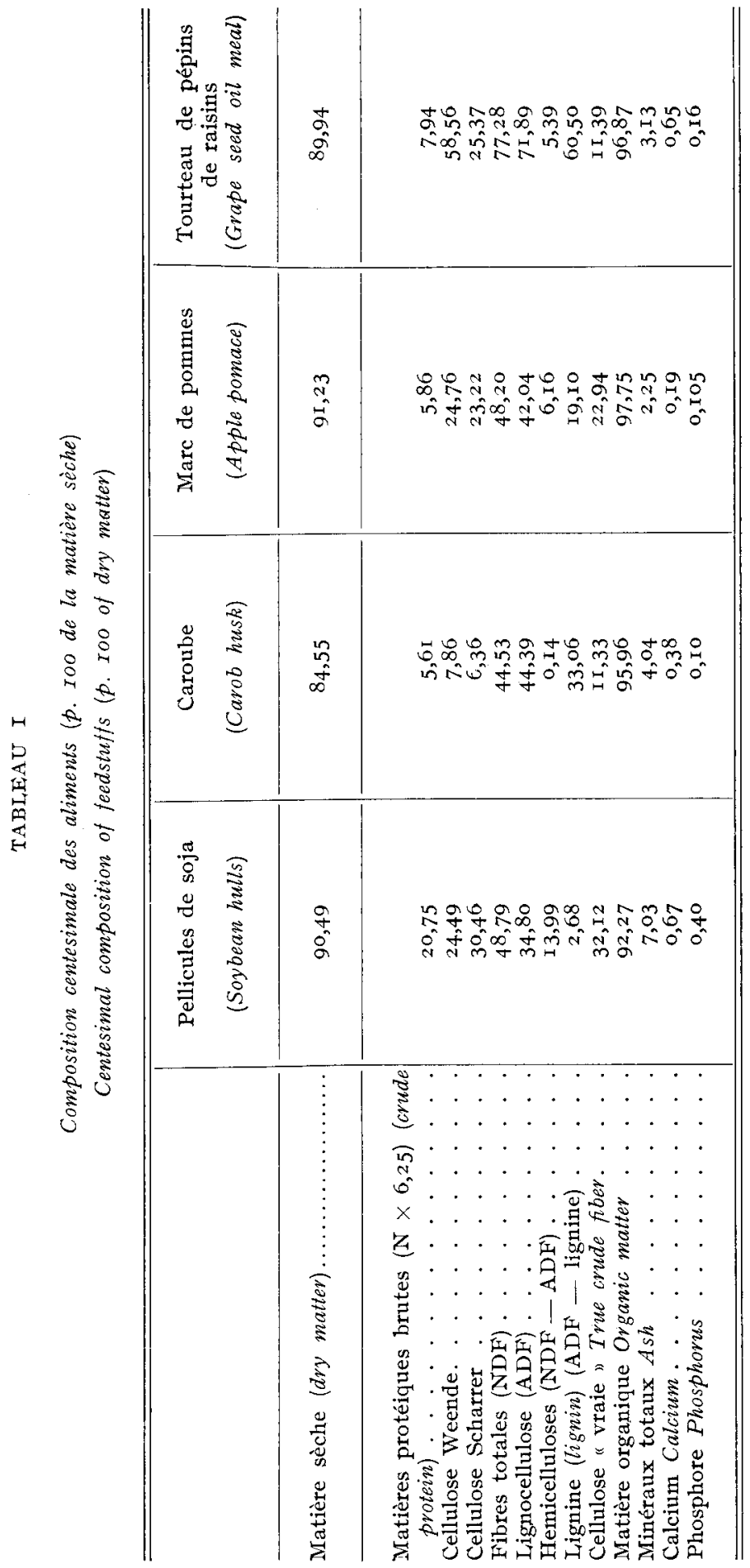




\section{II. - Résultats et discussion}

\section{A) Composition des aliments}

Pour un aliment donné, comme nous l'avions déjà constaté pour une précédente série d'aliments (Wor,TER et al., I 979), le tableau I vérifie une certaine similitude des taux de cellulose évalués selon les méthodes de Weende, de Scharrer ou de Van Soest ("cellulose vraie "), sauf dans le cas du tourteau de pépins de raisins dont la particulière richesse en lignine retentit sensiblement sur le résultat du dosage de Weende, alors que la teneur en "cellulose vraie " apparaît relativement faible.

La comparaison des 4 aliments étudiés fait d'ailleurs ressortir la nette infériorité du tourteau de pépins de raisins dont l'excès de lignine laisse prévoir une très mauvaise digestibilité. En revanche, les pellicules de soja se distinguent par leur faible degré de lignification, ainsi que par une teneur protéique quelque peu supérieure aux moyennes couramment citées (MICHALET, DorEaU et DEMARQUIL, Y, I 980 ), et surtout nettement plus élevée que pour les 3 autres matières premières, ce qui devrait aussi améliorer l'utilisation digestive apparente de l'azote.

\section{B) Coefficients de digestibilité}

Les résultats récapitulés aux tableaux 2 et 3 confirment la nette supériorité des pellicules de soja, par rapport aux caroubes ou au marc de pommes, et surtout par opposition au tourteau de pépins de raisins.

- Ainsi, la digestibilité de la matière organique atteint respectivement $0,7 \%$ $0,5^{8}-0,53$ et $0, \mathrm{I} 2$. De ce fait, les pellicules de soja sont assez comparables aux pulpes de betteraves; les caroubes et les mares de pommes prennent une place intermédiaire qui les rapprochent du son de blé ou de la farine de luzerne (WoLTER et al., I979); par contre, le tourteau de pépins de raisins ne vaut pas mieux que la paille de blé, et sa valeur énergétique doit être à peu près nulle chez les chevaux, alors qu'elle est estimée vers $0,24 \mathrm{UF} / \mathrm{kg}$ MS chez des ruminants (Cortryn et al., I979).

- Pour ces quatre aliments relativement riches en constituants membranaires (tabl. I), les différences de digestibilité globale, et vraisemblablement de valeur énergétique par voie de conséquence, s'expliquent essentiellement par les variations de l'utilisation digestive des fibres (tab1. 3), laquelle est très dépendante de la teneur en lignine. Ainsi celle-ci corrobore bien son tôle dépresseur de la digestibilité et donc de la valeur énergétique des aliments. A ce propos, 1'utilisation du tourteau de pépins de raisins ne peut se concevoir qu'à titre de lest, et de façon obligatoirement très restreinte pour ne pas déprimer la valorisation de l'ensemble de la ration, à l'inverse, des pellicules de soja, dont l'incorporation dans la ration améliore d'ailleurs sensiblement la digestibilité de la partie fibreuse chez les bovins (MACGREGOR et al., I976) comme chez le porc (KORNEGAY, I978).

Par ailleurs, dans cet essai, la digestibilité propre de la lignine reste comprise entre 5 et $7 \mathrm{p}$. roo, montrant que celle-ci peut encore servir de marqueur pour des mesures de digestibilité, si on admet une certaine approximation et à défaut de 
“ DIGESTIBILITÉ CHEZ LE PONEY "

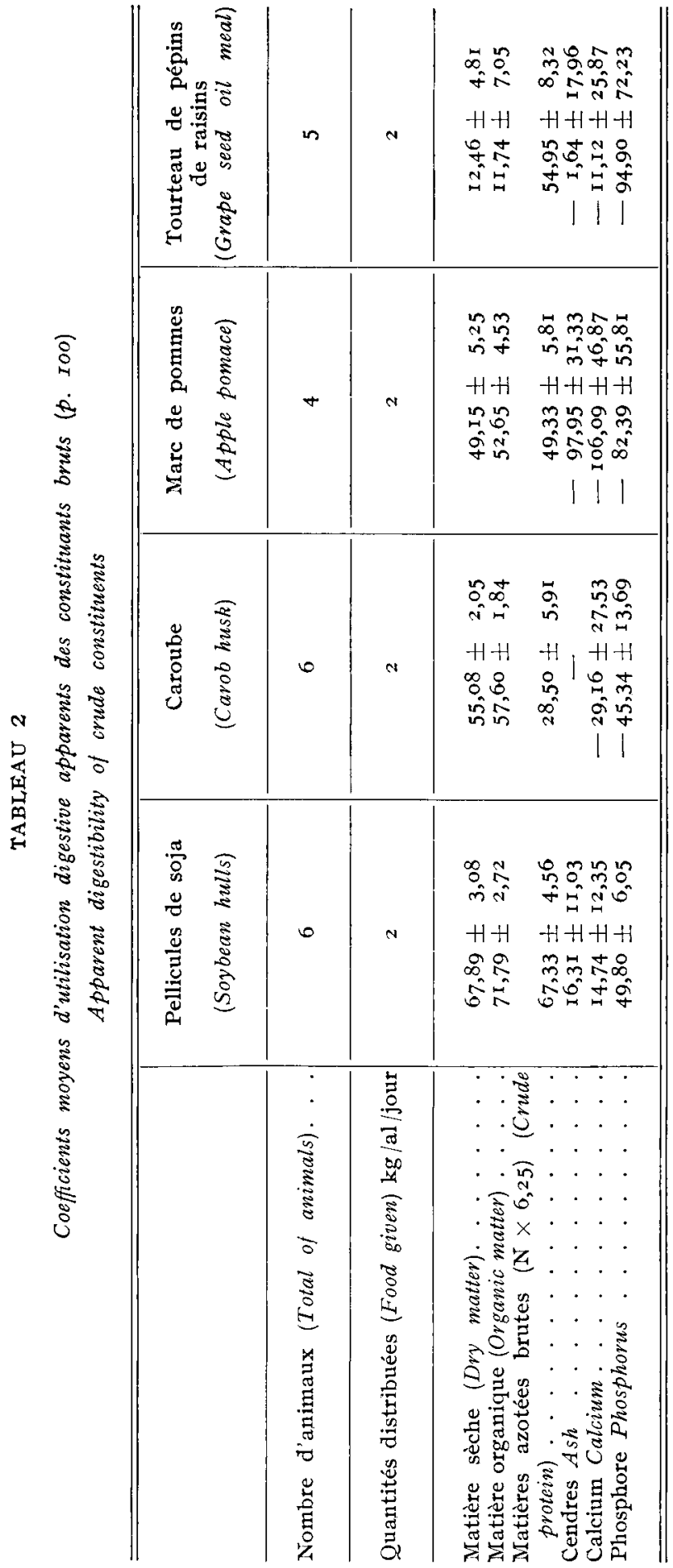




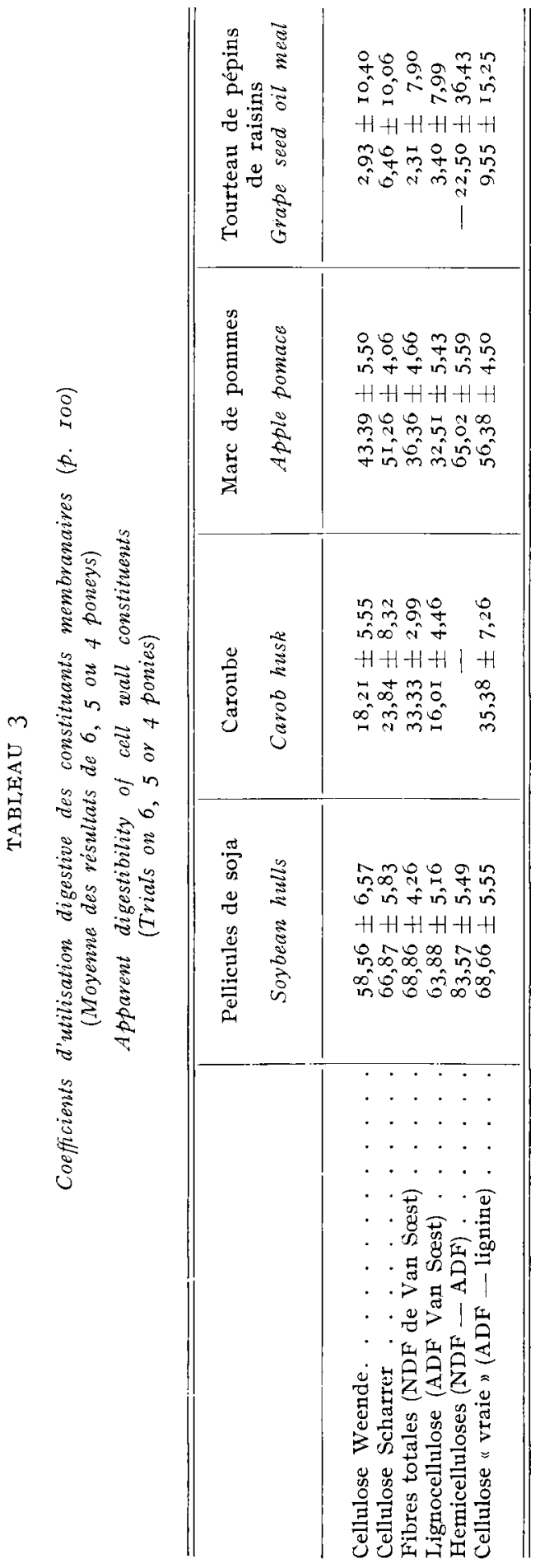


meilleure solution pratique. Toutefois, la lignine dosée par la méthode de Van SoEsT demeure assez mal définie et sa digestibilité apparente tient peut-être à l'imprécision du dosage.

Au contraire, il apparaît que les différentes autres fractions de glucides membranaires isolées par la méthode de Van SoEst (hémicelluloses, cellulose "vraie "), aussi bien que les fibres totales (N.J.F.), ont une digestibilité très inégale selon la source alimentaire considérée, aussi imprévisib!e que pour les celluloses brutes de WEENDE ou de ScharRer, démontrant, comme nous l'avions déjà mis en évidence lors de nos précédents essais (WoLTER et al., I979), qu'il ne s'agit pas d'entités chimiques bien précises, de valeur alimentaire déterminée.

- La digestibilité apparente des protéines, qui est d'abord tributaire du taux azoté (en plus de l'influence négative de la teneur en fibres brutes), est logiquement plus élevée avec les pellicules de soja. Parmi les trois autres matières premières, assez semblables par leur teneur azotée relativement faible, les caroubes se distinguent par une plus médiocre résorption protéique, éventuellement en rapport avec la présence de tanins. A plus forte raison, ceux-ci inhibent sans doute fortement la digestibilité de l'azote contenu dans le tourteau de pépins de raisins, déjà handicapés par sa richesse en lignine.

- De même, l'utilisation digestive apparente des minéraux est très mauvaise, sans doute parce que les apports alimentaires insuffisants compensent trés mal les pertes intestinales d'origine endogène, comme cela semble particulièrement manifeste avec le marc de pommes. Dans ce dernier cas, peut-être s'ajoute-t-i1 1'effet négatif de l'acide malique sur la digestibilité du calcium.

\section{C) Évolution de la glycémie}

Comme l'indique le tableau 4, la glycémie anteprandiale est avec le régime de marcs de pommes, comme lors de l'emploi d'aliments composés complets assez concentrés (WOLTER, DURIX et LETOURneau, I976), plus haute qu'avec les pellicules de soja, les caroubes ou le tourteau de pépins de raisins. A la suite du repas, elle atteint son maximum dans les 2 heures, comme avec les aliments complets (WOLTER et al., I978) et la progression relative apparaît nettement plus forte avec les caroubes, vraisemblablement en raison de leur richesse particulière en saccharose.

\section{Conclusion}

$A u$ total, il ressort de ces essais que les pelli,ules de soja, riches en cellulose facilement digestible même chez les équidés, est une bonne ssurce alimentaire pour ceux-ci, au même titre que les pulpes de betteraves, avec le bénéfice supplémentaire d'un apport azoté supérieur, en quantité et vraisemblablement en qualité.

Les caroubes et le marc de pommes ont une valeur intermédiaire, comparable à celle du son ou de la farine de luzerne, ce qui justifie également leur introduction dans les aliments composés.

Au contraire, le tourteau de pépins de raisins est une matière première très médiocre en raison de sa forte teneur en lignine, et son emploi doit être très restreint dans l'alimentation des chevaux. 


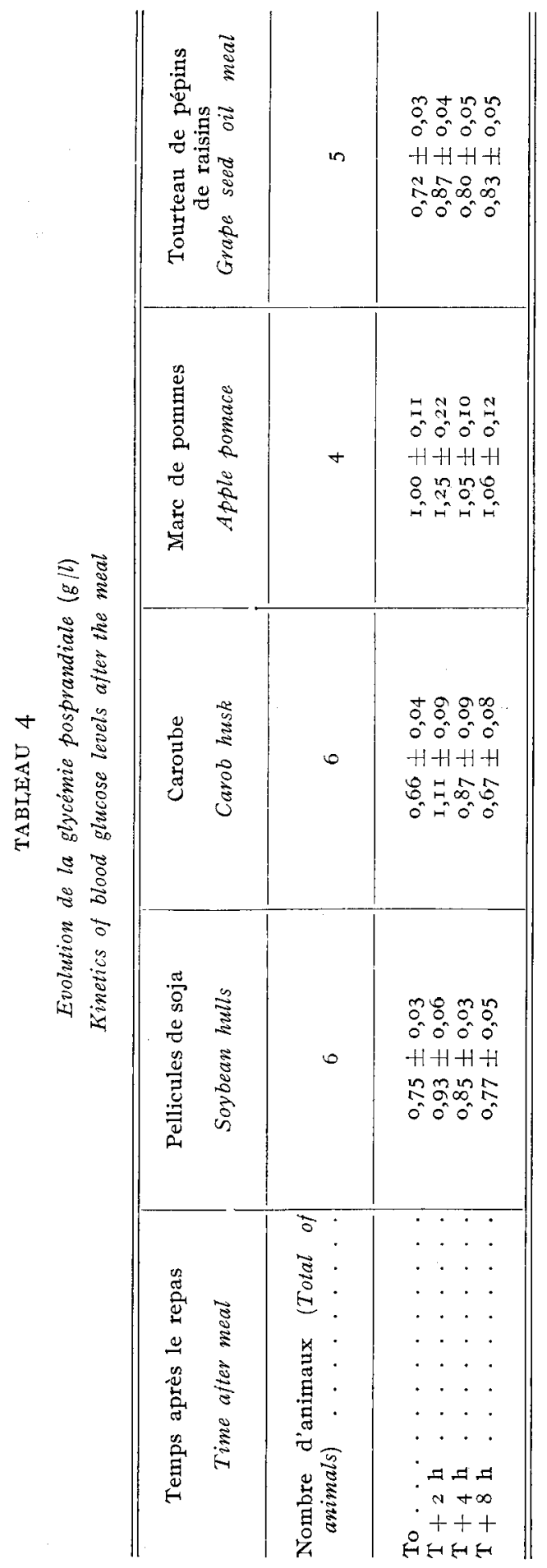




\title{
Summary
}

\author{
Estimation of total digestibility of soybean hulls, \\ apple pomace, carob husk, grape seed oil-meal.
}

Six or four ponies, placed in metabolism crates, were fed alternatively: soybean hulls, apple pomace, carob husk, grape seed oil meal for total digestibility trials $(2 \mathrm{~kg}$ feed/animal/day in two meals).

The cell wall constituents determined by the methods of Weende, Scharrer, and Van Soest, were similar, except for grape seed oil-meal, the rate of lignin of which was very high (tabl. I).

The nutritive value of soybean hulls, was comparable with dried sugar beet pulp. Carob and apple pomace were comparable with wheat bran and dried lucerne. The apparent digestibility of grape seed oil meal was not satisfactory (tabl. 2 and 3 ).

\section{Références bibliographiques}

CotTyn B. G., Boucque C. V., Aer's J. V., Buysse F. X., I979. Grape seed oil meal as cheap structural feedstuff in rations for intensive beef production. $Z$. Thierphysiol Tierernahr Futtermittelk, 41, 243-248.

Michalet-Dorfau B., DemarQuil. Y C., I980. Valeur alimentaire des pellicules de différentes graines oléagineuses. Bull. Tech. C.R.Z.V., Theix, INRA (39), I5-22.

Mrснег, M. C., г97г. Analyse quantitative de quelques substances azotees et glucidiques en milieu biologique. Thèse de Docteur d'Université. Université de Clermont-Ferrand.

Mac Gregor C. A., Owen F. G., Mac GILL D., I976. Effect of increasing ration fiber with soybean mill run on digestibility and lactation performance. $J$. Dairy Sci., 59, 682-689.

Kornegay E. T., I978. Feeding value and digestibility of soybean hulls for swine. J. Anim. Sci., 47 (6), I $272-\mathrm{I} 279$.

WOLTER R., Govy D., I 976 . Étude expérimentale de la digestion chez les équidés par analyse du contenu intestinal après abattage. Revue Méd. Vét., 127, I 723-I 736.

Wolter R., ChaAbouni A., I979. Étude de la digestion de l'amidon chez le cheval par analyse du contenu digestif après abattage. Revue Méd. Vét., 130 (10), I345-I357.

WOLter R., Andrée Durix, LETourneau J. C., I974. Influence du mode de présentation du fourrage sur la vitesse du transit digestif chez le poney. Ann. Zootech., 23, 293-242.

Wolter R., Andrée Durix, Letourneau J. C., I976. Influence du mode de présentation du fourrage sur la digestibilité chez le poney. Ann. Zootech., 24, 237-242.

WOLter R., Andrée DURix, LETOURneau J. C., I976. Influence du mode de présentation d'un aliment complet sur la vitesse du transit digestif et la digestibilité chez le poney. Ann. Zootech., 25, I 8 I - I 88 .

Wolter R., Gouy D., Andrée Durix, Letrourneau J. C., Monique Carceiten, Jacqueline IanDREAU, I978. Digestibilité et activité biochimique intracaecale chez le poney recevant un même aliment complet présenté sous forme granulée, expansée ou semi-expansée. Ann. Zootech., 27, 47-6o.

Wolter R., Andrée Durix, Letourneau J. C., Monique Carcerien, i 979. Évaluation chez le poney de la digestibilité du maïs-fourrage déshydraté, de la pulpe de betterave, de la luzerne déshydratée, du son de blé, de la paille de blé et des pulpes de raisins. Ann. Zootech., 28, 93-I oo. 